Bundesgesetzgebers über die Erforderlichkeit neuer Bundesgesetze der konkurrierenden Gesetzgebung beteiligt werden (S. 247 ff.).

Ob diese Vorschläge des Verfassers tauglich erscheinen, soll hier dahinstehen. Bekanntlich ist der verfassungsändernde Gesetzgeber 2006 mit der ersten Stufe der Föderalismusreform einen anderen Weg gegangen, indem er sich um die fühlbare Reduzierung der $\mathrm{Zu}$ stimmungsbedürftigkeit und die Rückführung von Gesetzgebungsmaterien in die Landesgesetzgebung bemüht hat. Die Bedeutung der Erforderlichkeitsprüfung hat er hingegen deutlich reduziert. Diese Entwicklungen behandelt die Arbeit, die offenbar 2005 abgeschlossen worden ist, nicht mehr. Das mindert ihre Relevanz für die aktuelle Diskussion, ohne dass ihr das hier entgegengehalten werden soll. Ärgerlich ist demgegenüber aber, dass im Umschlagstext und im Vorwort fälschlich der Eindruck erweckt wird, die Arbeit behandele die ,jüngste Föderalismusreform“. Das ist dann doch des Marketings zuviel.

Horst Risse

\title{
Deutscher Föderalismus: klare Sicht der Realitäten, problematische Propagierung von Wettbewerb
}

Baier, Christina: Bundesstaat und Europäische Integration. Die „Europatauglichkeit" des deutschen Föderalismus, Duncker \& Humblot, Berlin 2006, 323 Seiten, € 79,80.

Blankart, Charles B.: Föderalismus in Deutschland und in Europa, Nomos Verlagsgesellschaft (Bd. 1 der Neuen Studien zur politischen Ökonomie), Baden-Baden 2007, 218 Seiten, Є 29,-.

Föderalismus hat Konjunktur: Deutschland reformiert die Beziehungen von Bund und Ländern, und in bisher zentralistisch regierten Staaten nehmen Regionen mit Legislativbefugnissen auch an der Europapolitik teil. Für die deutschen Länder konstatiert Christina Baier in ihrer Münsteraner rechtswissenschaftlichen Dissertation jedoch: „Der deutsche Föderalismus hat sich als nicht europatauglich erwiesen“ (S. 285) - weder durch die Mitwirkung des Bundesrats an der Setzung von Gemeinschaftsrecht noch durch die der Länder an seiner Umsetzung. Keine Föderalismusreformkommission wird es ändern. Zu sehr stößt sich das gesamtstaatliche Interesse am Ego der Landesfürsten. Der Trennungsansatz von Charles B. Blankart, Professor für Volkswirtschaftslehre an Berlins Humboldt-Universität, käme dem entgegen; er erörtert aber die innerstaatlichen Finanzbeziehungen und will Wettbewerb der Gebietskörperschaften bis zur Insolvenz. Derweil Baier ihr Urteil mit der Praxis der Bundesrats- und Ländermitwirkung begründet, argumentiert Blankart enggeführt ökonomisch.

Baier geht von der parteipolitischen Praxis aus. Wenn die Mehrheiten „parteipolitisch divergieren, birgt (es) die Gefahr in sich, dass es zu Blockaden kommt, die eher auf der Parteiraison gegründet sind als auf europäischen Erwägungen" (S. 82). So will jede Bundestagsopposition für EGV-Änderungen eine Zweidrittelmehrheit im Bundesrat, nicht nur für Hoheitsrechtsübertragungen - 1994 die SPD bei der Norderweiterung, 2004 die CDU/ CSU bei der Osterweiterung. Bundestag und Bundesrat waren sich auch uneins beim Finanzrahmen für die Jahre 2007 bis 2013, als Berlin den Beitrag der Mitgliedstaaten auf ein 
Prozent des Bruttonationaleinkommens einfror, die Länder aber mehr Strukturförderungsmittel verlangten. Politisch wurde ein Streit vermieden, zumal sein Ausgang beim Bundesverfassungsgericht offen gewesen wäre. Wichtig ist es für Baier aber nur, wenn es um mehr geht als die Verringerung deutschen Einflusses.

Über EG-Vorhaben sind beide Kammern laut Art. 23 Abs. 2 GG frühestmöglich umfassend zu unterrichten. Die Bundesratsstellungnahme muss maßgeblich berücksichtigt werden, wenn eine Länderkompetenz berührt ist (dies beschränkte 2006 die Verfassungsreform), obwohl EG-Vorhaben sie meist nur zum Teil berühren. Bei politischen Konflikten - Baier nennt den EU-Beitritt der Türkei - darf der Bund davon auch zur Wahrung gesamtstaatlicher Verantwortung nicht abweichen: „Damit erhalten die Länder die Möglichkeit, die Verhandlungsposition des Bundes bei einem Regelungsvorhaben der Europäischen Gemeinschaft selbständig zu bestimmen und notfalls auch gegen die Auffassung der Bundesregierung durchzusetzen." (S. 45) Nicht erörtert wird von der Autorin die parlamentarische Kontrolle der Stellungnahmen und Mitwirkung eines Landesministers im EG-Ministerrat (Art. 203 EGV / Art. 23 Abs. 6 GG). Als Angehörige des Bundesorgans müssen die Mitglieder sich allenfalls politisch im Landtag rechtfertigen. Baier fragt nur, „ob das qualifizierte Mitwirkungsverfahren auch angesichts der dadurch entstehenden neuen Herausforderungen eine angemessene Form der Beteiligung der Bundesländer an der europäischen Gesetzgebung ist." (S. 92) Unvertretbar sei auch das Verfahren im Bundesrat. Wie der Bundestag kann er nicht jährlich 8000 EG-Dokumente behandeln. Der Bundesratsdirektor wählt dafür etwa 160 aus. Zumindest die großen Länder hätten aber durchaus die Verwaltungskapazität zur Prüfung der übrigen circa 7840 (S. 84).

Fast die Hälfte der Richtlinien setzen die Länder verspätet um. Je komplexer die Regelung ist, desto komplizierter sind die Abstimmungsprozesse zwischen Bund und Ländern. So kommt es „regelmäßig zu Fristversäumnissen, wenn die Länder an der Umsetzung in nationales Recht zu beteiligen sind, und die Fristüberschreitungen (sind) in diesen Fällen außerdem besonders groß“ (S. 150). Denn Europas mehreren Rechtstraditionen verpflichteter Gesetzgebung entspreche keine eindeutige deutsche Legislativkompetenz, die zersplittert, oft „versteinert“ sei. Häufig fällt es den Ländern auch schwer, „Konzepte zu akzeptieren, die in anderen Ländern entwickelt wurden und nicht ihren eigenen Vorstellungen entsprechen“; sie wollen „so viel wie nur möglich belassen wie bisher“ (S. 170 f.). Die Folgen: Vertragsverletzungsverfahren und Zwangsgeldandrohungen. Das Land, das „sich gern als Musterknabe der Integration präsentiert, (ist) bei der Anwendung des Gemeinschaftsrechts offenbar nicht in der Lage, (das) hohe integrationspolitische Credo in der Realität der nationalen Gesetzgebung zu beweisen" (S. 144). Baier schweigt aber zu den notwendigen parlamentarischen Sanktionen.

Ihr Fazit lautet: „Zusammenfassend ist festzuhalten, dass sich die Länderbeteiligung gemäß Art. 23 GG in der Praxis nicht bewährt hat." (S. 135) Die Föderalismuskommission sei beim Bemühen um die deutsche Europatauglichkeit an machtpolitischen Erwägungen gescheitert. Ihr gelang es nicht, „sich aus der Routine der Aushandlungsprozesse zwischen Parteien und zwischen Bundes- und Landesregierungen zu lösen" (S. 286). Vor allem starke Länder wie Nordrhein-Westfalen oder Bayern seien nicht bereit gewesen, dem Bund die Primärzuständigkeit in EU-Angelegenheiten zu überlassen. Die Länder sahen keinen Reformbedarf bei der Umsetzung von Richtlinien oder der Vertretung Deutschlands im Ministerrat durch einen ihrer Minister; es blieb beim Status quo. Für Deutschland als EUMitglied bedeute dies, „dass auch zukünftig Probleme der Länderbeteiligung gemäß Art. 23 
GG sowie der fristgerechten Umsetzung von EG-Richtlinien in nationales Recht ihre integrationspolitische Handlungsfähigkeit beeinträchtigen werden“ (S. 287).

Baier unterbreitet abschließend einen klaren Reformvorschlag: Kein Landesminister dürfe Deutschland mehr im Ministerrat vertreten, und die Bundesratsstellungnahme solle - wie beim Bundestag - nicht mehr bindend sein. Die EG-Dokumente müsse ein permanentes Bundesratsgremium beraten - ein Modell für die Landtage zur Kontrolle der Bundesratseuropapolitik? Überholt ist seit dem 28. August 2006 eine Richtlinienumsetzung durch Rahmengesetz mit Abweichungsrecht der Länder. Und die Idee vom Bundeszwang ist unrealistisch. Auch als nur Bremen die Kommunalwahlrichtlinie nicht umsetzen wollte, hätte der Bundesrat sie abgelehnt. Ein Mittel aber ist der von Baier angemahnte neue Art. 104a Abs. 6 GG über die Haftung für Zwangsgeld wegen der Nichtumsetzung einer Richtlinie.

Baiers Dissertation ist ein wertvolles Quellenwerk zur Ländermitwirkung. Im Juli 2005 beendet, nach Juli 2006 gedruckt ist sie aber teilweise nicht mehr aktuell. Das Kapitel zum Verfassungsvertrag aus Sicht der Länder - nur eine Fußnote nennt die Voten Frankreichs und der Niederlande - behandelt nicht den im Sommer 2006 absehbaren Reformvertrag; die Grundgesetzänderungen der Föderalismuskommission I traten im August 2006 in Kraft. Das Buch hätte aktualisiert werden müssen, auch wenn es dann nicht mehr die Dissertationsfassung darstellen würde (Promotionsordnungen sollten dergleichen ermöglichen).

„Zur Verwirklichung eines vereinten Europa wirkt die Bundesrepublik Deutschland bei der Entwicklung der Europäischen Union mit, die demokratischen, rechtsstaatlichen, sozialen und föderativen Grundsätzen und dem Grundsatz der Subsidiarität verpflichtet ist...", heißt es in Art. 23 Abs. 1 Satz 1 GG. Das entspricht der Bundesstaatsklausel in Art. 20 Abs. 1 GG, die durch Art. 79 Abs. 3 GG Ewigkeitsgeltung hat, da nicht nur die Gliederung des Bundes in Länder, sondern auch ihre grundsätzliche Mitwirkung bei der Gesetzgebung nicht berührt werden dürfen. Welche Probleme das für die Europapolitik bereitet, analysiert Baier. Sie ist nicht allein mit ihrer Kritik am real existierenden deutschen Föderalismus, der für die heute noch im Wesentlichen zentralistisch verfassten Staaten Europas kein nachahmenswertes Vorbild ist. Eine Gegenstimme im Sinne eines radikalen Föderalismus kommt von Charles B. Blankart.

Für ihn fördern nur föderale Vielfalt und Systemwettbewerb Freiheit, Rechtsstaatlichkeit und Kreativität: „Europa ist nicht durch politische Einheit, sondern durch seine Fraktionierung in eine Vielzahl politisch autonomer Territorien, Herrschaften und dergleichen erfolgreich geworden." (S. 13) In elf Kapiteln beschreibt er die Geschichte des Föderalismus in Deutschland und Europa, zeigt den deutschen Paradigmenwechsel nach 1919 zu mehr Zentralstaat und ausgabenfreudigeren Ländern und Gemeinden. Wichtig ist ihm ein Wettbewerb der Gebietskörperschaften ähnlich dem von Unternehmen auf dem Markt (S. 15). Die Finanzierung von Staatenbund und Bundesstaat erläutert er in je zwei Kapiteln für die EU und Deutschland. Dem folgen Regeln gegen opportunistisches Handeln in Gebietskörperschaften sowie Vorschläge zur Föderalismusreform II vor allem mit dem Ziel, dass die für ihre Haushaltswirtschaft selbstverantwortlichen Gebietskörperschaften Bund, Länder und Gemeinden ohne bündische Solidarität „pleite gehen“ können.

Blankart argumentiert an der Realität vorbei, hat einen eurozentrierten kulturellen Ansatz und behandelt weder die Identiät von Gebietskörperschaften noch die Emotion der Bewohner, die etwa in Leipzig am 27. Januar 2008 mit großer Mehrheit gegen den Verkauf öffentlichen Eigentums stimmten. Über 70 Prozent der deutschen Exporte gehen in den EG-Raum, das sind nahezu 50 Prozent des Bruttonationalprodukts; ähnlich sieht es bei 
allen EU- und EWR-Mitgliedstaaten aus. Das schreit geradezu nach dem Ende von Fraktionierung, denn nicht sie brachte „Europa in den vergangenen 3000 Jahren trotz zahlreicher Kriege so viel rascher voran als die meisten seiner Schwesterkulturen in den mittleren und östlichen Teilen Asiens" (S. 43), sondern das Christentum vor allem nach Reformation und Aufklärung. Und das kolonialistische westliche Denken dominierte die Weltwirtschaft, -kultur und -wissenschaft - wie zuvor arabische Kultur und Wissenschaft Europa geprägt hatten.

Aktuell sind die Ausführungen Blankarts zur Finanzverfassung: „Möglichst viele öffentliche Leistungen (sind) nach dem Prinzip der institutionellen Kongruenz zu organisieren, d.h. bei den Ländern Nutznießung, Entscheidung und Bezahlung zusammenzuführen.“ (S. 75) Dann gelte „unter dem Autonomieprinzip das Gesetz der Unsichtbaren Hand des Marktes“ (S. 179) - bis zur Zahlungsunfähigkeit mangels weiterer Kredite. Die „Aktiva (würden) in die Konkursmasse fallen und dadurch an die Gläubiger übergehen. Deren Verlust wäre eine empfindliche Strafe für die politische Führung (?) und somit ein effizienter ex-ante Schutz vor zu hohen Risiken in der Investitionspolitik“; zu den Aktiva gehörten auch die Kunstschätze in Stadt- und Landesmuseen. „Sicher würde ein Sturm der Entrüstung durch die Bevölkerung gehen, wenn beispielsweise wertvolle Handschriften oder ehemalige Kirchengüter zur Disposition gestellt würden. Aus ökonomischer Sicht ist dies aber nicht von zentraler Bedeutung." (S. 180) Die kulturelle Identität soll also zulasten der Bevölkerung der Ökonomie geopfert werden.

An zwei Kommunen spielt Blankart die Theorie durch: Leukerbad im Schweizer Kanton Wallis, das zahlungsunfähig die Kredite nicht mehr bedienen konnte und dem das Berner Bundesgericht mit Urteil vom 3. Juli 2003 innerstaatliche Solidarität verweigerte, und Niederoderwitz in Sachsen, wo der Bundesgerichtshof das Land im Urteil vom 12. Dezember 2002 mangels ausreichender Kommunalaufsicht in Haftung nahm. In Leukerbad trugen zwar die Gläubiger das Insolvenzrisiko - die Folgen für die Bevölkerung nennt Blankart aber nicht. In „Niederoderwitz sollten die Gemeindebürger keine autonome Finanzverantwortung treffen, sondern wie eine Stiftung überwacht werden“ (S. 177), ohne massive Folgen für die Menschen. In der völlig unrealistischen Annahme, sie könnten in Haushaltsfragen wirksam „ihre Regierung kontrollieren und gegebenenfalls selbstverantwortlich abwählen“ (S. 174) will Blankart vom Prinzip der Bundestreue zum „Konzept des Föderalismus mit beschränkter Haftung “ übergehen, um das seit der Weimarer Zeit gewachsene bündische Solidarprinzip durch Selbstverantwortlichkeit in Art. 109 Abs. 1 GG zu ersetzen: „Bund und Länder sind in ihrer Haushaltswirtschaft selbständig, selbstverantwortlich und voneinander unabhängig." (S. 165) Zudem sei $\$ 12$ Abs. 1 Insolvenzordnung ersatzlos zu streichen und die Beschränkung der Zwangsvollstreckung bei Bund, Ländern und Gemeinden ( $\$$ 882a ZPO) aufzuheben. Sie sollen insolvent werden können - mit unübersehbaren, nicht erörterten Folgen für die Menschen und die kulturelle Identität des Gemeinwesens. In Europa sei „die Autonomie im Bereich der Grundrechte ganz in der europäischen Föderalismustradition“ (S. 104), da die Wertegemeinschaft „dem Systemwettbewerb und der Kreativität entgegensteht“ (S. 96). Wo aber wären die Mitgliedstaaten heute - zumal ohne den Europäischen Gerichtshof, den er nicht erwähnt - in Sachen Frauengleichberechtigung, Diskriminierungsverbote, Verbraucher- und Umweltschutz, Kartellkontrolle...?

Die Entwicklung überholt auch Blankart. Die Föderalismuskommission II wird die bündische Solidarität bewahren, was schmerzhafte Eingriffe in Bestand oder Autonomie eines Landes nicht ausschließt; der Reformvertrag wird Europas Institutionen und Politiken wei- 
ter voranbringen, die Wertegemeinschaft und mit dem Europäischen Parlament die Demokratie stärken. Unter dem Eindruck der US-Finanzkrise wird die Einheit gefestigt werden zulasten des von Blankart gefürchteten natürlichen Zentralismus. „Durch das Nebeneinander kooperativer Herrschaften entsteht Systemwettbewerb, der friedlich oder kriegerisch sein kann.“ (S. 195) Hoffentlich meint er das nicht ernst.

Der deutsche Föderalismus ist das Ergebnis machtpolitischer äußerer Einwirkungen. 1866 konnte Preußen noch die mit Österreich verbündeten Mitgliedstaaten des Deutschen Bundes annektieren und zu Provinzen degradieren, wie es zu gleicher Zeit im Siegeszug von Sardinien-Piemont Ministerpräsident Conte di Cavour tat. Schon 1867 beim Norddeutschen Bund, noch mehr 1871 musste Otto von Bismarck im nun vereinten kleindeutschen Reich den Bundesstaaten erhebliche Rechte lassen. Die europäischen Mächte hätten einen deutschen Zentralstaat nicht zugelassen. Nach einer Unitarisierungsphase in der Weimarer Republik und dem NS-Zentralismus waren es wieder äußere Mächte, diesmal die westlichen Besatzer, die einen deutschen Föderalismus durchsetzten. Von vornherein war er auf eine Mischung der Gewalten angelegt. „Nicht absolute Trennung, sondern gegenseitige Kontrolle, Hemmung und Mäßigung der Gewalten ist dem Verfassungsaufbau des Grundgesetzes zu entnehmen." 1 Welche Probleme dies im europäischen Mehrebenensystem verursacht, schildert Baier. Wie sehr gegenseitige Abhängigkeit besteht, zeigen die Verhandlungen der zweiten Föderalismuskommission. An eine Trennung, gar eine absolute, wie sie Blankart vorschwebt, ist nicht nur nicht zu denken - es würde diese Staaten sprengen.

Erich Röper

1 BVerfGE 34, S. 52 ff., S. 59.

\section{Verfassungsgericht und Politik: Modellschreinerei statt „politischer“ Analyse, Methodenkompetenz als Handicap}

Hönnige, Christoph: Verfassungsgericht, Regierung und Opposition. Die vergleichende Analyse eines Spannungsdreiecks, VS Verlag für Sozialwissenschaften, Wiesbaden 2007, 280 Seiten, $€ 34,90$.

Wenn es zutreffen sollte, was Bertolt Brecht seinem „Galilei“ in den Mund legt, dass „das einzige Ziel der Wissenschaft darin besteht, die Mühseligkeit der menschlichen Existenz zu erleichtern" (14. Szene), dann hätte die hier zu rezensierende Potsdamer Dissertation auf grandiose Weise ihr Ziel verfehlt. Sie macht es nämlich weder den Juristen, die sich von Amts wegen mit dem Thema „Verfassungsgericht“ zu befassen haben, noch jenen Sozialwissenschaftlern, die wenigstens randständig an diesem Thema interessiert sein sollten, leicht, in ihrer wissenschaftlichen Existenz befestigt, gar in ihren Literatur-Interessen befriedigt zu werden, von einem zu erwartenden „politischen“ Mehrwert der Lektüre ganz zu schweigen. Im Gegenteil: Sie verlangt jedem Leser ein hohes Maß an asketischer Selbstdisziplin ab, sollte der sich überhaupt dazu durchringen, bis zur letzten Seite durchzuhalten. Am Ende wird er sich fragen, was er an Einsichten in die Sache, gar grundlegenden Erkenntnissen zum Verhältnis von Regierung, Opposition und Verfassungsgericht wirklich hinzugewon- 